\title{
Cirurgia bariátrica: atuação do enfermeiro na qualidade de vida pós-cirurgia
}

\author{
Bariatric surgery: nurse's role in post-surgery quality of life \\ Cirugía bariátrica: papel del enfermero en la calidad de vida posquirúrgica
}

Recebido: 26/01/2022 | Revisado: 04/02/2022 | Aceito: 06/02/2022 | Publicado: 12/02/2022

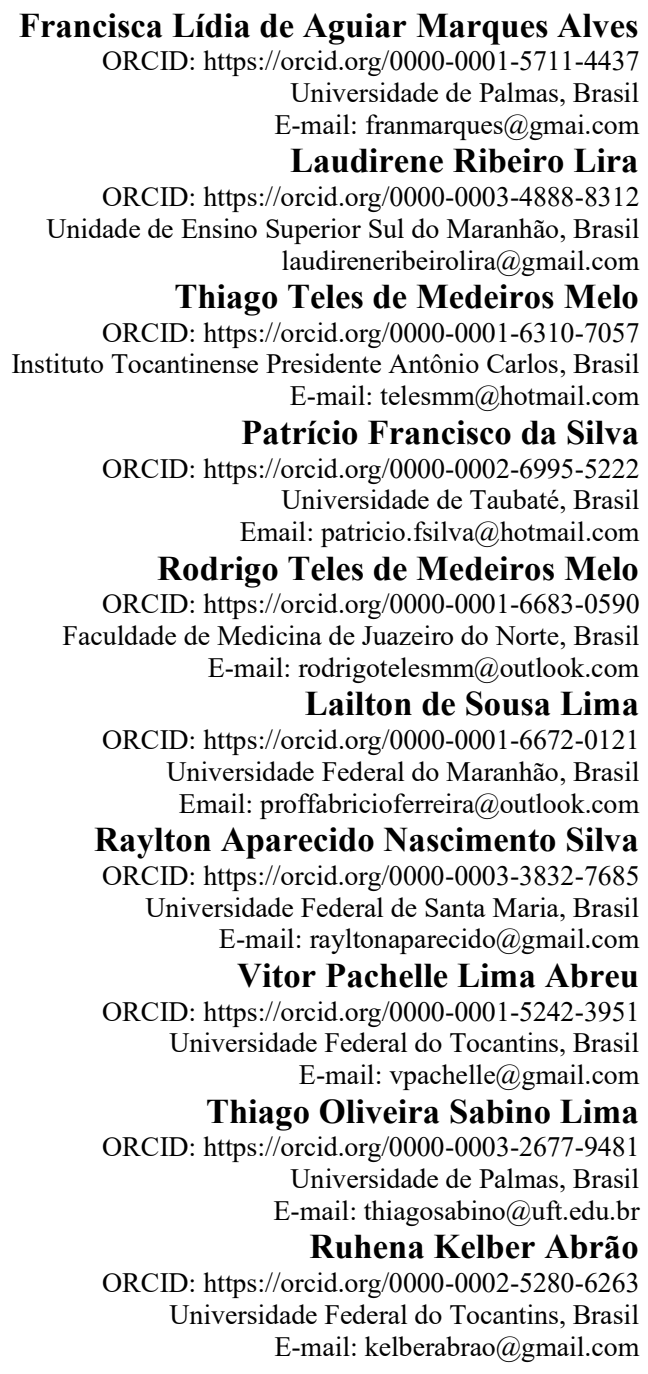

\begin{abstract}
Resumo
O Processo de Enfermagem segue um protocolo que se divide em cinco etapas: investigação, diagnóstico, planejamento, implementação e avaliação da assistência de enfermagem. É uma atividade exclusiva do Enfermeiro, profissional responsável por direcionar todo trabalho desenvolvido por sua equipe de enfermagem. O objetivo de este estudo baseia-se em uma análise da literatura o processo de cuidado de enfermagem aos pacientes em pré e pós-operatório de cirurgia bariátrica e ressaltar a notoriedade das políticas públicas de humanização na assistência prestada ao pacientes em pré e pós-operatório de cirurgia bariátrica. A metodologia está ancorada em uma abordagem qualitativa, recorrendo ao emprego de pesquisa bibliográfica. Os resultados e discussões demonstraram que esse é um processo que merece atenção por considerar que o acompanhamento de enfermagem auxilia na adaptação do paciente pós-cirurgia, minimiza as complicações e consequentemente influencia na recuperação do mesmo. Este estudo é necessário para demais pesquisas que viabilizem estratégias educativas para o profissional enfermeiro e estímulos à boa prática em assistência à saúde.
\end{abstract}

Palavras-chave: Cuidado de enfermagem; Bariátrica; Saúde.

\section{Abstract}

The Nursing Process follows a protocol that is divided into five stages: investigation, diagnosis, planning, 
implementation and evaluation of nursing care. It is an exclusive activity of the Nurse, the professional responsible for directing all the work developed by their nursing team. The objective of this study is based on an analysis of the literature on the nursing care process for patients in the pre and postoperative period of bariatric surgery and to highlight the notoriety of public policies for humanization in the care provided to patients in the pre and postoperative period. of bariatric surgery. The methodology is anchored in a qualitative approach, resorting to the use of bibliographic research. The results and discussions showed that this is a process that deserves attention because it considers that the nursing follow-up helps in the adaptation of the patient after surgery, minimizes complications and consequently influences his recovery. This study is necessary for other researches that enable educational strategies for the professional nurse and encourage good practice in health care.

Keywords: Nursing care; Bariatric; Cheers.

\section{Resumen}

El Proceso de Enfermería sigue un protocolo que se divide en cinco etapas: investigación, diagnóstico, planificación, ejecución y evaluación de los cuidados de enfermería. Es una actividad exclusiva de la Enfermera, profesional responsable de dirigir todo el trabajo desarrollado por su equipo de enfermería. El objetivo de este estudio se basa en un análisis de la literatura sobre el proceso de atención de enfermería a los pacientes en el pre y postoperatorio de cirugía bariátrica y resaltar la notoriedad de las políticas públicas para la humanización en la atención a los pacientes en el pre y postoperatorio. período de cirugía bariátrica. La metodología está anclada en un enfoque cualitativo, recurriendo al uso de la investigación bibliográfica. Los resultados y discusiones mostraron que este es un proceso que merece atención porque considera que el seguimiento de enfermería ayuda en la adaptación del paciente después de la cirugía, minimiza las complicaciones y consecuentemente influye en su recuperación. Este estudio es necesario para otras investigaciones que posibiliten estrategias educativas para el profesional de enfermería y fomenten la buena práctica en el cuidado de la salud.

Palabras clave: Atención de enfermería; Bariátrico; Salud.

\section{Introdução}

A Lei no 7.498 de 25 de junho de 1986 no seu Art. 11 destaca que o Enfermeiro exerce todas as atividades de enfermagem, cabendo-lhe privativamente: "cuidados de enfermagem de maior complexidade técnica e que exijam conhecimentos de base científica e capacidade de tomar decisões imediatas" (Brasil, 1986). Diante de tal abordagem podese afirmar que os cuidados de Enfermagem devem ocorrer "antes, durante e após" qualquer procedimento, não sendo diferente no caso de pacientes que se submetem ao procedimento da cirurgia bariátrica (Rocha et al, 2020).

Ressalta-se que a obesidade tem sido tratada e difundida no cotidiano de saúde na perspectiva biomédica. Sendo, portanto, entendida "como um agravo de caráter multifatorial envolvendo desde questões biológicas às históricas, ecológicas, econômicas, sociais, culturais e políticas", motivo pelo qual se constitui um desafio às práticas dos profissionais de saúde (Brasil, 2006; Sales et al, 2020).

De acordo com uma reportagem de Adauto Versiani, Presidente da Sociedade Brasileira de Endocrinologia e Metabologia - Regional Minas Gerais (SBEM-MG), publicada no "Portal o Tempo" no cenário mundial, considerando o evento da pandemia da covid-19 houve um aumento da obesidade no mundo, como sendo o reflexo das alterações que ocorreram na dinâmica da vida das pessoas, integrando questões de ordem econômica, social e, principalmente, cultural. Devido a estes fatores, é necessário um olhar abrangente reconhecendo os múltiplos aspectos relacionados à obesidade.

A reportagem traz ainda informações sobre as causas da obesidade e do sobrepeso, dentre as quais estão: a disponibilidade de alimentos com elevada densidade energética, ou seja, ricos em gordura e o estilo de vida sedentário. À medida que o peso aumenta, podem surgir uma série de doenças crônicas, como hipertensão, diabetes, colesterol alto, doenças cardiovasculares (infarto, derrame), doenças osteoarticulares e apneia do sono, podendo acelerar o processo de envelhecimento, sendo que a patologia ainda compromete a autoestima e a sociabilização (Rodrigues et al, 2020).

É oportuno destacar ainda que a obesidade é uma doença universal, sendo um dos principais problemas de saúde pública e considerada a segunda causa de morte que pode ser evitada, perdendo apenas para o tabagismo. Cerca de $20 \%$ dos brasileiros são obesos e 55\% estão acima do peso. A incidência da obesidade é maior em quem tem menos de 40 anos, e a internação requer mais cuidados pessoais, aparelhagem e dificuldade de movimentação. Cerca de $30 \%$ das pessoas estão 
com sobrepeso e 50\% das obesas estão internadas por causa de alguma complicação (Brasil, 2020).

Diante dessa realidade, entende-se que o cuidado de enfermagem dispensado ao paciente que foi submetido à cirurgia bariátrica se configura como fundamental. Deste modo, o questionamento que se faz é: O profissional de enfermagem está preparado técnica e psicologicamente para o atendimento ao paciente que foi submetido à cirurgia bariátrica?É pertinente destacar que o atendimento realizado pelos profissionais de enfermagem, assim como a atuação da equipe multidisciplinar faz total diferença no atendimento realizado nas Unidades Básicas de Saúde, assim como em todas as instituições que prestam atendimento à saúde. Contudo ainda é desafiador, considerando que o enfermeiro precisa estar preparado técnica e psicologicamente para realizar um acompanhamento de forma satisfatória (Gomes, Ferreira, Rodrigues et al, 2020).

Para direcionar o estudo, definiu-se como objetivo a análise ancorada na literatura bibliográfica o processo de cuidado de enfermagem aos pacientes em pré e pós- operatório de cirurgia bariátrica a fim de propiciar, mais especificamente, reflexões na área acadêmica e proporcionar estímulo de saber para melhorar a assistência frente a essa patologia.

\section{Revisão Bibliográfica}

\subsection{Caracterização de cirurgia bariátrica}

A obesidade é uma doença complexa, distinguida pelo acúmulo excessivo de gordura no organismo, que pode desencadear algumas doenças crônicas como a diabetes tipo II, dislipidemias, hipertensão arterial, coronariopatias, doenças articulares, apnéia do sono, cálculos biliares e alguns tipos de câncer (Aourn, 2014).

Dessa forma, o conceito de Gastroplastia, também chamada de cirurgia bariátrica, cirurgia da obesidade ou ainda de cirurgia de redução do estômago, é, literalmente, a plástica do estômago (gastro = estômago, plastia = plástica) que tem como o objetivo reduzir o peso de pessoas com o IMC muito elevado. Outro conceito pode ser atribuído e consiste em ser um procedimento que reúne um conjunto de técnicas de diminuição do estômago destinado à redução de peso de pacientes com obesidade (Sbcbm, 2020)

Diante desses conceitos, a cirurgia bariátrica se constitui como sendo um dos principais tratamentos para a diminuição de peso e consequentemente da melhoria das comorbidades associadas à obesidade. No Brasil, o progresso das técnicas cirúrgicas e a popularização da cirurgia originaram a expansão dos cuidados de enfermagem para essa clientela (Negrão, 2016).Considerando os riscos associados ao excesso de gordura corporal, a obesidade é considerada com um grande problema de saúde pública, que envolve todas as faixas etárias, independente do grupo socioeconômico, seja em países desenvolvidos quanto em desenvolvimento, com implicações sociais e psicológicas graves e de proporções desastrosas (Brasil, 2014).

É pertinente afirmar que de acordo com a Organização Mundial de Saúde (2016) a obesidade é apontada como a doença crônica que mais aumenta em prevalência no mundo, estimando-se que em 2025, o número de adultos com sobrepeso chegará a 2,3 bilhões, e de obesos a 700 milhões (Brasil, 2014). De acordo com as Diretrizes Brasileiras de Obesidade da Associação Brasileira para o Estudo da Obesidade e da Síndrome Metabólica (ABESO),a classificação mais usual para a obesidade é o Índice de Massa Corpórea (IMC), onde a regra é dividir o peso corporal pela altura (kg)/altura²). O resultado se subdivide em categorias, conforme se segue: Um IMC com resultado $(<18,5)$ é considerado de baixo peso, IMC de (18,5 - 24,9) peso normal, IMC de (25,0-29,9) sobrepeso, IMC de (30,0-34,9) está na categoria de obeso I, o de $(35,0-39,9)$ obeso II e de IMC $(\geq 40.0)(9,10)$ obeso III (Abeso. 2016).

O tratamento da obesidade é considerado multidisciplinar, ou seja, deve haver a intervenção de vários profissionais, combinando muitas vezes a terapia medicamentosa com mudanças no hábito alimentar e atividade física. A cirurgia 
bariátrica em muitos casos tem sido um recurso consistente para reverter o sobrepeso, contudo se faz necessário observar quais as indicações para esse tratamento, considerando que nem todas as pessoas têm essa indicação (Rêgo, 2017).

Conforme publicação da Associação Brasileira para o Estudo da Obesidade e da Síndrome Metabólica (2016), o paciente indicado para o procedimento da cirurgia bariátrica deve ter entre 18 a 65 anos, IMC acima de $40 \mathrm{~kg} / \mathrm{m}^{2}, \mathrm{IMC}$ acima de $35 \mathrm{~kg} / \mathrm{m}^{2}$ se tiver alguma comorbidade grave que esteja relacionado com a obesidade, deve apresentar algum documento de comprovação que o paciente não conseguiu ou não foi capaz de reduzir o peso, mesmo tendo acompanhamento durante pelo menos dois anos, incluindo a dietoterapia, psicoterapia, que realizou atividade física e fez uso de medicamento (Abeso, 2016).

Faz-se necessário destacar que o paciente com indicativo para cirurgia bariátrica, deve ter um acompanhamento sistematizado de uma equipe bem estruturada de profissionais, dentre os quais estão: médicos especialistas (endocrinologista e cirurgião bariátrico), nutricionista, psicólogo ou psiquiatra, anestesista, assistente social, cardiologista e, sem dúvida, o enfermeiro que deverá participar desde a fase pré-operatória até a alta hospitalar (Santos, 2016).Diante do que foi apresentado, cabe aos profissionais de saúde desenvolver um trabalho sistematizado no sentido de desenvolver estratégias capazes de mobilizar os obesos mórbidos para a adoção e manutenção de comportamento saudável, antes e após a realização do procedimento cirúrgico.

\subsection{Cuidados de enfermagem no acompanhamento ao paciente obeso e pós-cirúrgico de bariátrica}

O termo cuidado procede do latim cogitare e significa pensado, imaginado, mediato. A origem etimológica cogitátus, refletido. O cuidado também pode se referir à dedicação, aparência, formação moral e intelectual, atenção especial, comportamento precavido, zelo, desvelo que se dedica a alguém ou algo (Silva, et al 2010).

Nesse contexto apesar da importância do tema e dos mais variados interesses dos países quanto ao tema obesidade, a atuação da Enfermagem nessa área ainda está direcionada para as orientações médicas, visto o número reduzido de publicações realizadas em nosso país, voltadas especificamente, para a assistência de enfermagem prestada ao paciente submetido à cirurgia bariátrica (Felix, et al. 2012).

Tavares (2010) entende que o enfermeiro tem um papel muito importante durante o tratamento e na recuperação, pois o enfermeiro é quem irá ajudá-lo a reconhecer suas fraquezas, e, em função delas, constata que pacientes obterão sucesso ou não na cirurgia. É compreendido que o enfermeiro deverá fazer novas implementação, aderindo às dificuldades de cada paciente. É imprescindível que o enfermeiro esteja sempre alerta a qualquer alteração do quadro clínico do paciente cirúrgico, sabendo de sua atuação e seja capaz de intervir quando necessário (Oliveira et al, 2020).

Nesse aspecto, é pertinente afirmar que o enfermeiro deve participar de todo processo de atendimento ao paciente obeso. Este profissional tem a responsabilidade de ter conhecimento teórico e prático do tratamento proposto, se inteirar de todas as informações pré, trans e pós-operatórias, no intuito de prestar uma assistência de qualidade para que o resultado seja o mais satisfatório possível (Oliveira et al, 2020). Para tanto, o enfermeiro deve conhecer as propostas cirúrgicas, as vantagens e desvantagens da cirurgia, o desencadear de cada uma delas, para que os cuidados de enfermagem sejam estruturados conforme a necessidade para a recuperação pós-operatória do paciente (Negrão, 2016). Ainda segundo o autor acima citado o enfermeiro deve planejar e implementar estratégias que possibilitem a efetiva participação do paciente e família para a promoção do sucesso da cirurgia. Ele é responsável, 24 horas por dia, da orientação, do cuidado e até do apoio para que esse paciente consiga superar as fases, muitas vezes intensa e desgastante a que o paciente será submetido (Negrão, 2016).

Conforme Brunner et al. (2011), a assistência de enfermagem durante o período pós-operatório imediato concentrase em intervenções destinadas a prevenir ou tratar complicações, considerando que este é um momento crítico para o 
paciente e se faz necessário uma constante observação cuidadosa para manter as funções fisiológicas vitais dentro dos parâmetros da normalidade, até que os efeitos da anestesia desapareçam. Por menor que seja a cirurgia, o risco de complicações sempre estará presente, por esse motivo a prevenção destas, no pós-operatório promove rápida recuperação, reduz tempo, gastos, preocupações, ameniza a dor e aumenta a sobrevida.

A recuperação da Cirurgia Bariátrica é um processo lento que requer mudanças de estilo de vida para ser bemsucedida. Essas alterações devem estender-se ao ambiente familiar e ao local de trabalho, onde o apoio é crucial para o sucesso global do tratamento contra a obesidade (Dungan, 2018).

Destaca-se nesse entendimento que o pós-operatório é uma fase nova onde se inicia uma rotina extremamente diferente, considerando que deve haver uma adaptação ao novo estilo de vida. Dessa forma, o enfermeiro atua de maneira que o paciente se recupere conforme esperado, sem complicações e seguindo todas as prescrições médicas. Esse processo transoperatório é o início de uma grande mudança na vida do paciente, é onde inicia toda mudança e o acompanhamento de um profissional capacitado são de fundamental importância (Deram, 2018).

Tanaka, et al. (2013) afirmam que:

O enfermeiro atua diretamente na recuperação do paciente, para restabelecer o imediato equilíbrio fisiológico, auxiliando-o para que possa seguir adequadamente as orientações médicas e muitas vezes fazendo por ele algo que ele não consiga fazer, como por exemplo, o seu autocuidado. Também é papel do enfermeiro acompanhar esse paciente, em suas pequenas rotinas, tornando assim mais seguro e eliminando os possíveis riscos ao paciente.

É pertinente afirmar que os inconvenientes pelos quais os pacientes pós cirúrgicos de cirurgia bariátrica passam, inclui dentre outras complicações o suicídio, sendo considerados em números significativo. Luz, (2012), afirma que de dois a cada cinco pacientes cometeram suicídio no terceiro mês de cirurgia. Na maioria dos casos isso incide em decorrência da falta de preparação ou acompanhamento do paciente por profissionais capacitados, considerando as mudanças que o procedimento causa.

Dessa forma, está mais do que comprovado que o enfermeiro exerce papel fundamental no preparo desse paciente para tais mudanças. É responsabilidade de esse profissional explicar de forma clara e segura o que o espera no pósoperatório de cirurgia bariátrica, bem como orientar aos familiares, tornando mais simplificada a adaptação de ambos. Normalmente, não é a fome que torna essa adaptação difícil, pois com os líquidos a fome é saciada, mas a ilusão de que a necessidade de mastigar pode desenvolver sentimentos como arrependimento e frustração no paciente (Felix, 2012).

A ligação criada entre o enfermeiro e o paciente em qualquer que seja o procedimento se constitui como o melhor caminho para uma boa recuperação, considerando que na enfermagem o paciente encontra muito mais que só o apoio para o seu autocuidado. É importante que se estabeleça uma relação de confiabilidade entre profissional e paciente para garantir a sua reabilitação com sucesso e consequentemente possa alcançar o objetivo que se espera, sua total reabilitação (Luz, 2012).

\section{Metodologia}

Este estudo constitui-se de uma revisão da literatura do estilo narrativa que de acordo com Rother (2007) é um método que possui como objetivo a realização de estudos a partir de pesquisas já publicadas sobre a mesma temática, de forma ordenada, sistemática e abrangente, possibilitando uma informação mais ampla acerca do assunto estudado. A mesma foi realizada de agosto a dezembro de 2020, no qual foram realizadas consultas a livros, periódicos e em artigos científicos selecionados por meio da busca nos bancos de dados da Scielo, publicações de revistas de saúde, portarias do Ministério da Saúde, dentre outros (Schwartz et al, 2020).

A busca nos bancos de dados foi realizada utilizando terminologias cadastradas nos Descritores em Ciências da Saúde pela Biblioteca Virtual em Saúde desenvolvidas a partir do Medical Subject Headings da U.S. National Library of 
Medicine, que permite o uso da terminologia comum em português, inglês e espanhol. As palavras chaves utilizadas na busca foram: "Cuidado de enfermagem", "Bariátrica”, "Qualidade de vida”. Foram considerados como critérios de seleção: (a) Texto completo da publicação disponível; (b) procedência nacional; (c) publicações relacionadas ao período de 2010 a 2020, bem como documentos oficiais, como leis e resoluções que estão em vigor, independente da data de publicação (d) conteúdo relacionado atuação do enfermeiro na qualidade de vida pós-cirurgia bariátrica; (e) idioma português. Sendo então desconsiderados os textos que não contemplaram os critérios a, b, c, d, e, e supracitados.

\section{Resultados e Discussão}

Para fundamentar os resultados e discussão, foi utilizada à síntese dos textos pesquisados, a fim de buscar uma melhor compreensão sobre a Cirurgia Bariátrica: Atuação do Enfermeiro na Qualidade de Vida Pós-Cirurgia, sendo esse procedimento de grande valia, haja vista que contribui de forma significativa para uma aprendizagem baseada em evidências, além de favorecer a aquisição do conhecimento e permitir que o profissional de enfermagem possa colocá-los em prática por meio do que foi aprendido (Silva et al, 2021).

Considerou-se para estruturação deste estudo os textos de idioma português que foram publicados entre os anos de 2010 a 2020, conforme os critérios de exclusão e inclusão. Assim, constatamos que os resultados obtidos nas bases de dados por meio da filtragem foram significativos como demonstrado na figura 1.

Figura 1. Fluxograma da amostragem dos artigos obtidos nas bases de dados SciELO; Biblioteca Virtual de Saúde; Revistas de Saúde. Palmas (TO), Brasil (2020).

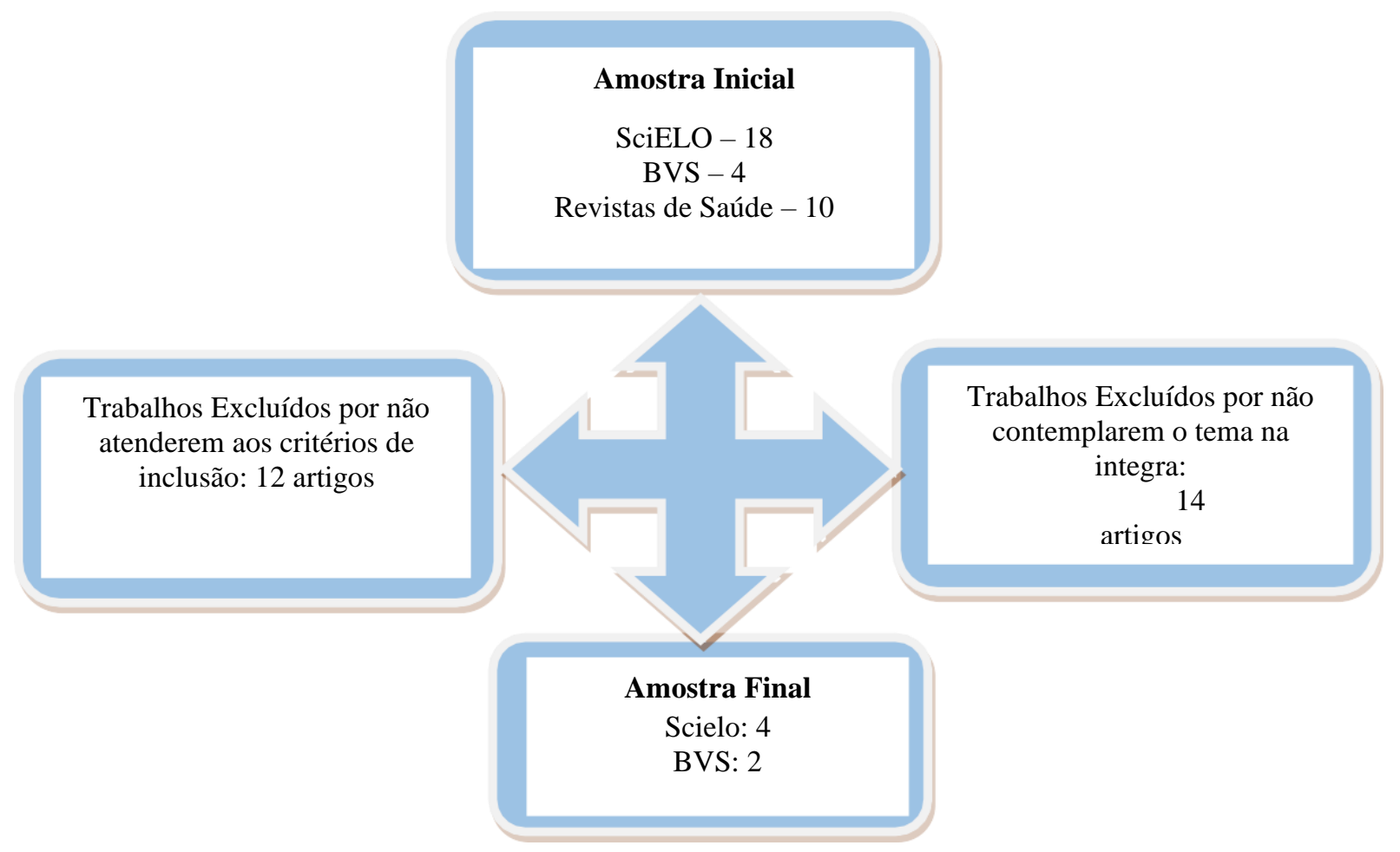

Fonte: Autores (2020).

Para melhor compreensão dos textos, foi feita uma análise sistematizada onde contemplam: o título, autor, objetivo, metodologia e os resultados encontrados, conforme o Quadro 1. 
Quadro 1: Quadro sinóptico dos artigos selecionados.

\begin{tabular}{|c|c|c|c|c|c|}
\hline Título & Autor (es) & Ano & Objetivos & Metodologia & Resultados \\
\hline $\begin{array}{c}\text { Cuidados de } \\
\text { enfermagem no } \\
\text { pós operatório de } \\
\text { cirurgia } \\
\text { bariátrica }\end{array}$ & $\begin{array}{l}\text { Santos; } \\
\text { Camilo }\end{array}$ & 2016 & $\begin{array}{c}\text { Descrever a } \\
\text { qualidade dos } \\
\text { serviços de } \\
\text { enfermagem } \\
\text { prestados às pessoas } \\
\text { no pós-operatório de } \\
\text { cirurgia bariátrica }\end{array}$ & $\begin{array}{l}\text { Estudo de revisão } \\
\text { que se estende além } \\
\text { da esfera biológica; }\end{array}$ & $\begin{array}{l}\text { Experiência mais próxima da realidade } \\
\text { que envolve obesidade e qualidade de vida } \\
\text { aos submetidos à cirurgia. }\end{array}$ \\
\hline $\begin{array}{l}\text { Assistência de } \\
\text { enfermagem ao } \\
\text { paciente } \\
\text { submetido à } \\
\text { gastroplastia: um } \\
\text { estudo } \\
\text { bibliográfico }\end{array}$ & $\begin{array}{l}\text { Marques; } \\
\text { Silva }\end{array}$ & 2015 & $\begin{array}{l}\text { Analisar a } \\
\text { popularização da } \\
\text { gastroplastia no } \\
\text { Brasil; }\end{array}$ & $\begin{array}{c}\text { Revisão } \\
\text { bibliográfica }\end{array}$ & $\begin{array}{lccr}\text { Estudo demonstra } & \text { diagnóstico } & \text { e } \\
\text { intervenções para a } & \text { assistência de } \\
\text { Enfermagem voltada } & \text { ao paciente } \\
\text { submetido à gastroplastia } & & \end{array}$ \\
\hline $\begin{array}{l}\text { A enfermagem } \\
\text { no atendimento } \\
\text { pós-cirurgia } \\
\text { bariátrica - } \\
\text { revisão } \\
\text { sistemática da } \\
\text { literatura }\end{array}$ & $\begin{array}{c}\text { Leonor } \\
\text { Cipitelli, et al }\end{array}$ & 2019 & $\begin{array}{l}\text { Identificar } \\
\text { estratégias de } \\
\text { prática clínica da } \\
\text { enfermagem a } \\
\text { pacientes } \\
\text { submetidos à } \\
\text { cirurgia bariátrica. }\end{array}$ & $\begin{array}{l}\text { Pesquisa descritiva e } \\
\text { qualitativa de } \\
\text { abordagem } \\
\text { bibliográfica }\end{array}$ & $\begin{array}{l}\text { O estudo ressalta a importância da } \\
\text { participação efetiva da enfermagem no } \\
\text { diagnóstico, no cuidado e na orientação do } \\
\text { paciente submetido à cirurgia bariátrica } \\
\text { e, principalmente, nos cuidados intensivos } \\
\text { durante a recuperação tardia. }\end{array}$ \\
\hline $\begin{array}{c}\text { Protocolo de } \\
\text { assistência de } \\
\text { enfermagem ao } \\
\text { paciente em pré e } \\
\text { pós-operatório de } \\
\text { cirurgia } \\
\text { bariátrica }\end{array}$ & $\begin{array}{c}\text { Felix et } \\
\text { al }\end{array}$ & 2012 & $\begin{array}{l}\text { Identificar os } \\
\text { indicadores } \\
\text { empíricos para as } \\
\text { demandas } \\
\text { terapêuticas de } \\
\text { autocuidado. }\end{array}$ & $\begin{array}{l}\text { Pesquisa de } \\
\text { abordagem } \\
\text { exploratória }\end{array}$ & $\begin{array}{l}\text { O protocolo de assistência é um } \\
\text { importante recurso para instrumentalizar } \\
\text { as orientações prestadas pelos enfermeiros } \\
\text { ao paciente bariátrico, visando ao } \\
\text { autocuidado, além de dar subsídios para } \\
\text { sistematizar a assistência de enfermagem a } \\
\text { ser prestada. }\end{array}$ \\
\hline $\begin{array}{c}\text { Cirurgia } \\
\text { bariátrica por } \\
\text { bypass gástrico } \\
\text { em Y de Roux: } \\
\text { abordagem da } \\
\text { técnica e de } \\
\text { possíveis } \\
\text { complicações } \\
\text { tardias no pós- } \\
\text { operatório. } \\
\end{array}$ & $\begin{array}{l}\text { Rodrigues et } \\
\text { al. }\end{array}$ & 2019 & $\begin{array}{c}\text { Identificar a } \\
\text { importância da } \\
\text { assistência de } \\
\text { enfermagem ao } \\
\text { paciente submetido } \\
\text { a bariátrica, e o } \\
\text { acompanhamento no } \\
\text { período } \\
\text { transoperatório. }\end{array}$ & $\begin{array}{l}\text { Pesquisa de } \\
\text { abordagem } \\
\text { exploratória }\end{array}$ & $\begin{array}{l}\text { É fundamental o acompanhamento } \\
\text { de uma equipe multidisciplinar, não } \\
\text { deixando de incluir o papel do enfermeiro, } \\
\text { que é fundamental na recuperação do } \\
\text { paciente e no apoio da família do paciente } \\
\text { bariátrico }\end{array}$ \\
\hline $\begin{array}{l}\text { Perda de peso e } \\
\text { sua associação } \\
\text { com indicadores } \\
\text { metabólicos no } \\
\text { pós-operatório de } \\
\text { cirurgia } \\
\text { bariátrica }\end{array}$ & Cassiano et al. & 2018 & $\begin{array}{l}\text { Compreender o } \\
\text { cuidado dos } \\
\text { profissionais de } \\
\text { enfermagem às } \\
\text { pessoas que se } \\
\text { submetem à cirurgia } \\
\text { para redução de } \\
\text { peso. }\end{array}$ & $\begin{array}{c}\text { Revisão } \\
\text { bibliográfica }\end{array}$ & $\begin{array}{l}\text { Os resultados deste estudo contribuíram } \\
\text { para a compreensão do cuidado de } \\
\text { enfermagem como um cuidado que se } \\
\text { relaciona à própria dimensão sociocultural } \\
\text { do ser que cuida, o profissional de } \\
\text { enfermagem. }\end{array}$ \\
\hline
\end{tabular}

Fonte: Autores (2020).

Após a apreciação dos artigos, realizou-se uma análise comparativa entre os textos selecionados, no sentido de apresentar uma abordagem sobre o cuidado de enfermagem dispensado ao paciente pós-cirurgia bariátrica, considerando o impacto ocasionado na vida de cada paciente submetido a esse procedimento, sendo organizada conforme se segue.

Os autores Santos (2016), Gesiel (2015) e Cipitelli, et al (2019), são unânimes em afirmar que a cirurgia bariátrica é uma das alternativas mais eficazes para a perda de peso é ainda o principal tratamento para a redução de peso e a melhora das comorbidades associadas à obesidade.

No aspecto que trata sobre a assistência de enfermagem ao paciente que será ou foi submetido à cirurgia bariátrica, o Cofen apud Felix (2012, p. 86) descreve o protocolo técnico e um instrumento normativo do processo de intervenção técnica e social que orienta os profissionais na realização de suas funções. Tem como base os conhecimentos científicos e práticos do cotidiano do trabalho em saúde de acordo com cada realidade. Nesse protocolo, são definidos como requisitos para o autocuidado referente à oxigenação, hidratação, alimentação, eliminação, atividades, sono e repouso, solidão e interação social, prevenção de riscos à vida e ao bem estar, promoção da saúde, requisito de autocuidado de 
desenvolvimento, requisito de autocuidado nos desvios de saúde. Ressalta-se que este protocolo está fundamentado na Teoria de Autocuidado de Orem.

Rodrigues (2019) e Cassiano (2018) enfatizam a importância do acompanhamento ao paciente, no pré, trans e após a cirurgia bariátrica, evidenciando que o enfermeiro é o elo facilitador entre os demais profissionais e familiares, minimizando as dúvidas e medos relacionados à cirurgia, tendo papel fundamental na orientação do paciente, assim contribuindo para a boa adesão ao tratamento da obesidade e consequentemente pela recuperação e sucesso da cirurgia.As autoras ainda comungam do pensamento de Pires (2019), o qual afirma que o cuidar em enfermagem deve sempre buscar "promover a vida, o potencial vital, o bem-estar dos seres humanos na sua individualidade, complexidade e integralidade".

\section{Considerações Finais}

A redução do peso é um processo lento e gradativo que provoca sensações de desânimo e frustrações, considerando que comer o que se deseja é uma forma prazerosa de viver para muitas pessoas. Contudo, esse prazer pode significar um tormento quando o indivíduo não consegue controlar seus impulsos e desejos, desenvolvendo assim um processo patológico, a obesidade.

Dessa forma, o auxílio de profissionais se manifesta como um suporte fundamental para alcançar os objetivos que a pessoa obesa determina para a redução do peso.Assim, de acordo com os textos pesquisados, a equipe multiprofissional se manifesta como fator determinante para que o procedimento da cirurgia bariátrica, visualizada como um dos principais procedimentos para minimizar os riscos e agravos decorrentes do aumento de peso.

Em especial, se destaca o enfermeiro pois este é o profissional que realiza o acompanhamento do paciente desde a Atenção Básica, também chamada de Atenção Primária, como sendo a porta de entrada para o atendimento, dando continuidade na atenção secundária e terciária, para garantir que o atendimento seja satisfatório e alcance os resultados esperados.

É pertinente afirmar que a concretização desta pesquisa foi de grande valia, sendo possível entender o peso do desempenho do enfermeiro frente aos pacientes que vivenciaram a cirurgia bariátrica.Dessa forma, é de extrema importância que a equipe multidisciplinar esteja presente, pois durante esse processo todos se tornam fundamentais no enfrentamento, transição e adaptação ao processo de cirurgia bariátrica, sendo estes profissionais os que passam o maior tempo no cuidado deste paciente durante todo processo.

Contudo, conpreende-se que há uma deficiência em relação a capacitação técnica dos profissionais de enfermagem para o cuidado a esses pacientes, considerando que na maioria dos casos não há diferenciação do cuidado realizado com as outras patologias.A capacitação profissional é sempre bem vinda em todos os procedimentos e se faz necessário, diante das várias situações adversas que podem surgir no pré, trans e pós-cirúrgico de bariátrica.

Assim, a conclusão que se faz é que apesar de ser um tema bastante atualizado existem poucas informações com base científica na literatura disponível, embora a adesão ao método de redução do estômago pelos pacientes tenha aumentado verticalmente.

Diante de toda abordagem instituída em relação ao tema, compreende-se grande possibilidade para a atuação do enfermeiro no contexto de cuidados ao paciente submetido à cirurgia bariátrica, considerando ser um tema que está em evidência e permite que esse profissional possa atuar de forma abrangente, haja vista que os avanços tecnológicos permitem a atualização quanto à capacitação para a oferta do cuidado personalizado e que atenda as especificidades de cada paciente. 


\section{Referências}

Associação de enfermeiros registrados no perioperatório (Aorn). Diretriz de cirurgia bariátrica. Aourn 2014; 79 (5): $1026-52$

Brasil, Sociedade Brasileira de Cirurgia Bariátrica e Metabólica. (2020). O que é Obesidade? http://www.endocrino.org.br/o-que-e-obesidade/.

Brasil. “- Lei n 7.498/86, de 25 de junho de 1986 Conselho Federal de Enfermagem - Brasil.” Conselho Federal de Enfermagem, 25 June 1986, http://www.cofen.gov.br/lei-n-749886-de-25-de-junho-de-1986_4161.html. Accessed 2020.

Brasil. Ministério da Saúde. Obesidade. Cadernos de Atenção Básica - $\mathrm{n}^{\circ} 12$ [Internet]. Brasília: Ministério da Saúde; 2006. http://189.28.128.100/dab/docs/publicacoes/cadernos_ab/abcad12.pdf.

Brasil. Ministério da Saúde. Vigilância de fatores de risco e proteção para doenças crônicas por inquérito telefônico (Vigitel 2014) http://www.abeso.org.br/uploads/downloads/80/553a243c4b9f3.pdf.

Brunner \& Suddarth, Tratado de enfermagem médico-cirúrgica /(2011) [editores] Suzanne C. Smeltzer...[et al.] ; [revisão técnica Isabel Cristina Fonseca da Cruz, Ivone Evangelista Cabral ; tradução Antonio Francisco Dieb Paulo, José Eduardo Ferreira de Figueiredo, Patricia Lydie Voeux].: Guanabara Koogan, 2011. 2v. : il. ; $21 \times 28 \mathrm{~cm}$.

Cassiano, R., et al. "13. Perda de peso e sua associação com indicadores metabólicos no pós-operatório de cirurgia bariátrica | Cassiano | Revista Científica UMC.” Open Journal Systems, 2018, http://seer.umc.br/index.php/revistaumc/article/view/245.

Cipitelli, Leonor, and Isabel Cruz. "Nursing Evidence -based inteprofissional practice guidelines for care in post-bariatric surgery - ICU - Systematic Literature Review. | Spala | Journal of Specialized Nursing Care.” Journal of Specialized Nursing Care, 2019, http://www.jsncare.uff.br/index.php/jsncare/article/view/3180/806.

Deram, Sophie. (2018). “O que é cirurgia bariátrica: entenda melhor este processo”, Sophie Deram PhD.

Diretrizes Brasileiras de Obesidade. Abeso. 2016, $4^{\text {a }}$ Edição. Disponível em: http://www.abeso.org.br/diretrizes.

Dungan, S. (2018). Parte II: retorno ao trabalho após a cirurgia bariátrica. Aaohn Journal, 56(12)

Felix et al; (2012). Protocolo de assistência de enfermagem ao paciente em pré e pós-operatório de cirurgia bariátrica, Revista Brasileira de Enfermagem, Brasília, 65(1), 83-91.

Gomes, A. V.., Ferreira, R. K. A.., \& Rodrigues, C. F. do C. (2020). A saúde na vida do cárcere no Brasil e no Tocantins. Research, Society and Development, 9(9), e981998067. https://doi.org/10.33448/rsd-v9i9.8067

Luz et al. (2012). Vantagens e desvantagens da cirurgia bariátrica para o tratamento da obesidade mórbida. RBONE-Revista Brasileira de Obesidade, Nutrição e Emagrecimento, 2(10).

Marques, G., \& Silva, D. (2016). “Assistência de enfermagem ao paciente submetido à gastroplastia: um estudo bibliográfico." Fema, 2015, https://cepein.femanet.com.br/BDigital/arqTccs/1111370439.pdf. Accessed 2020.

Negrão. R. J. S., \& Bianchi, E. R. F. A atuação do enfermeiro na assistência prestada ao paciente submetido à cirurgia bariátrica. Prát Hos;8(44):145-8.

Oliveira, L. L. S. de, Lima, T. O. S. ., Silva, R. A. N., Silva, R. M. O. ., Abreu, V. P. L. ., \& Ferreira, R. K. A. . (2020). Atuação do enfermeiro na assistência a mulher com câncer de ovário . Research, Society and Development, 9(9), e43996962. https://doi.org/10.33448/rsd-v9i9.6962

Pires D. (2019). A enfermagem enquanto disciplina, profissão e trabalho. Rev Bras En- ferm [Internet]. 62(5):739-44. http://dx.doi.org/10.1590/S003471672009000500015 .

Rêgo AS, Zulin A, Scolari S, Marcon SS, Radovanovic Cat. (2020) Análise das condições clínicas de pessoas obesas em período pré e pós-operatório de cirurgia bariá- trica. Ver Col Bras Cir 2017; 44(2): 171-178. www.scielo.br/pdf/rcbc/v44n2/pt 0100-6991-rcbc-44-02-00171.

Rocha, C. C., Lima, T. S., Silva, R. A. N., \& Abrão, R. K. (2020). Abordagens sobre sífilis congênita. Research, Society and Development, 9(8), e984986820. https://doi.org/10.33448/rsd-v9i8.6820

Rodrigues, C. F. do C., Alves, M. M. M., Brustulin, R., \& Ferreira, R. K. A. (2020). Avaliação do Controle do HIV/Aids na Atenção Primária em Palmas/TO. Research, Society and Development, 9(9), e372997126. https://doi.org/10.33448/rsd-v9i9.7126

Rodrigues, Rayane, et al. (2019) "Cirurgia bariátrica por bypass gástrico em Y de Roux: abordagem da técnica e de possíveis complicações tardias no pósoperatório." Cirurgia bariátrica por bypass gástrico em Y de Roux: abordagem da técnica e de possíveis complicações tardias no pós-ope, 2019, https://acervomais.com.br/index.php/cientifico/article/download/4979/3574/.

Rother, Edna. (2007) "Brasil - Revisão sistemática X revisão narrativa Revisão sistemática X revisão narrativa.” SciELO, 2007, https://www.scielo.br/j/ape/a/z7zZ4Z4GwYV6FR7S9FHTByr/?lang=pt.

Sales, O. P., Vieira, A. F. B., Martins, A. M., Garcia, L. G., Ferreira, R. K. A. O Sistema Único de Saúde: desafios, avanços e debates em 30 anos de história. Humanidades \& Inovação. Tocantins, 6(17), 54-65.

Santos, M. B. P., \& Camilo, J. C. (2016). Cuidados de Enfermagem no pós-operatório de cirurgia bariátrica. Simpósio de TCC e Seminário de IC. $2^{\circ}$.

SBEM. “congremem 2019 - SBEM.” Sociedade brasileira de endocrinologia e metabologia, https://www.endocrino.org.br/congremem-2019/.

Schwartz, S., Vieira, M. A.., Rodrigues, A. C. S., \& Ferreira, R. K. A. (2020). Estratégias para o trabalho com textos na universidade. Research, Society and Development, 9(8), e790986209. https://doi.org/10.33448/rsd-v9i8.6209 
Research, Society and Development, v. 11, n. 3, e5311326214, 2022

(CC BY 4.0) | ISSN 2525-3409 | DOI: http://dx.doi.org/10.33448/rsd-v11i3.26214

Silva, I. et al. Cuidado, autocuidado e cuidado de si: uma compreensão paradigmática para o cuidado de enfermagem. Revista da escola de enfermagem da USP, 43(3), 697-703, 2010

Silva, M. S. da ., Gomes, T. B. ., Silva, R. A. N. ., Almeida, N. M. ., Ribeiro, T. B. ., Lima, T. O. S. de ., Rosa, C. M. ., \& Ferreira, R. K. A. . (2021). Síndrome de Burnout em Profissionais de Enfermagem. Research, Society and Development, 10(12), e409101220747. https://doi.org/10.33448/rsd-v10i12.20747

Sociedade Brasileira de Cirurgia Bariátrica e Metabólica. (2020) https://www.sbcbm.org.br/.

Tanaka, Denise Spósito, Peniche, Aparecida de Cássia Giani. (2009). Assistência ao paciente obeso mórbido submetido à cirurgia bariátrica: dificuldades do en- fermeiro. Acta Paulista de Enfermagem, 22(5).

Tavares et al; (2010). Obesidade e qualidade de vida: revisão da literatura. Revista Médica de Minas Gerais. 20(3), $359-366$. 